\title{
Tuberculosis Presenting as an Inflammatory Pseudotumor of the Sciatic Nerve in a Rheumatoid Arthritis Patient Taking Etanercept
}

Bruno Miguel Fernandes(D, MD, Rheumatology Department, Centro Hospitalar Universitário São João; José Miguel Pereira, MD, Radiology Department, Centro Hospitalar Universitário São João; Pedro Rodrigues Pereira, MD, Pathology Department, Centro Hospitalar Universitário São João; Miguel Bernardes, MD, PhD, Rheumatology Department, Centro Hospitalar Universitário São João, Porto, Portugal. Address correspondence to Dr. B.M. Fernandes, Alameda Prof. Hernâni Monteiro, 4200-319 Porto, Portugal. Email: bfernandesmg@gmail.com. The authors declare no conflicts of interest relevant to this article. Ethics approval was granted by the authors' institution and the patient's informed consent was obtained.

Active tuberculosis (TB) in patients treated with anti-tumor necrosis factor- $\alpha$ (TNF- $\alpha$ ) agents usually results from the reactivation of a latent infection..$^{1,2}$

A 46-year-old woman with rheumatoid arthritis in Disease Activity Score in 28 joints remission who was taking etanercept (ETN) was admitted in our hospital due to a 4-month history of progressive hypoesthesia and paresthesia, initially on her left foot and later with extension up to the left knee.

The neurological examination revealed decreased muscle strength, particularly in the left foot dorsiflexion. Electromyography showed postganglionic changes in nerve conduction, affecting motor and sensory fibers, with signs of

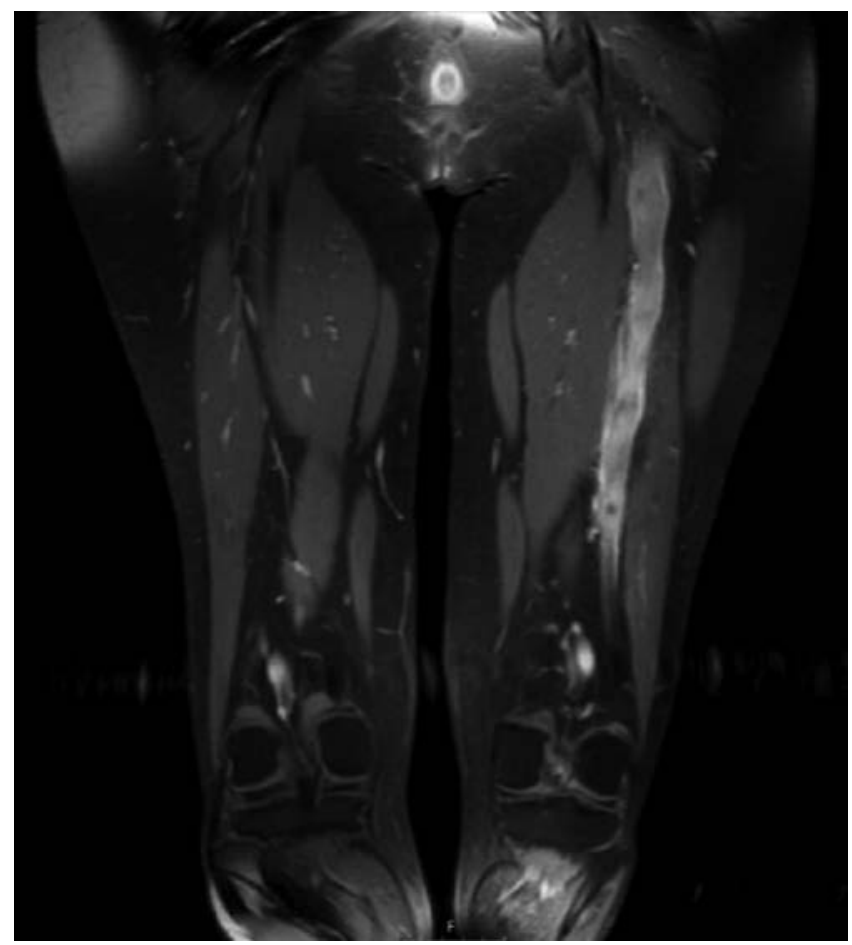

Figure 1. Coronal T1-weighted fat-saturated gadolinium-enhanced image of the thighs, showing fusiform thickening along the longest axis of the left sciatic nerve with diffuse heterogeneous contrast enhancement with an approximate length of $22 \mathrm{~cm}$ and maximum transverse diameter of $1.6 \mathrm{~cm}$. subacute and severe denervation of L5-S1 myotome-dependent muscles, suggesting a proximal lesion above the sciatic trunk. Lumbar spine magnetic resonance imaging (MRI) was irrelevant, and MRI of the thighs showed a fusiform left sciatic nerve mass with contrast enhancement (Figure 1).

Histology of the left sciatic nerve biopsy showed a lymphoplasmacytic inflammatory infiltrate, with necrotic epithelioid granulomas and multinucleated giant cells, with no signs of malignancy (Figure 2). These features, despite the negative Ziehl-Neelsen staining, associated with a positive tuberculin test (negative tuberculin test previously to ETN), led to a diagnosis of TB of the sciatic nerve sheath and the suspension of this anti-TNF- $\alpha$ agent; treatment with isoniazid, rifampicin, pyrazinamide, and ethambutol was initiated. After 9 months of treatment and a rehabilitation program, the patient showed significant clinical and imaging improvement.

In countries with high incidence of $\mathrm{TB}$, it is recommended to screen and treat latent $\mathrm{TB}$ prior to anti-TNF therapy. ${ }^{3}$ Extrapulmonary TB may arise, even without evidence of lung involvement, as this rare case described.

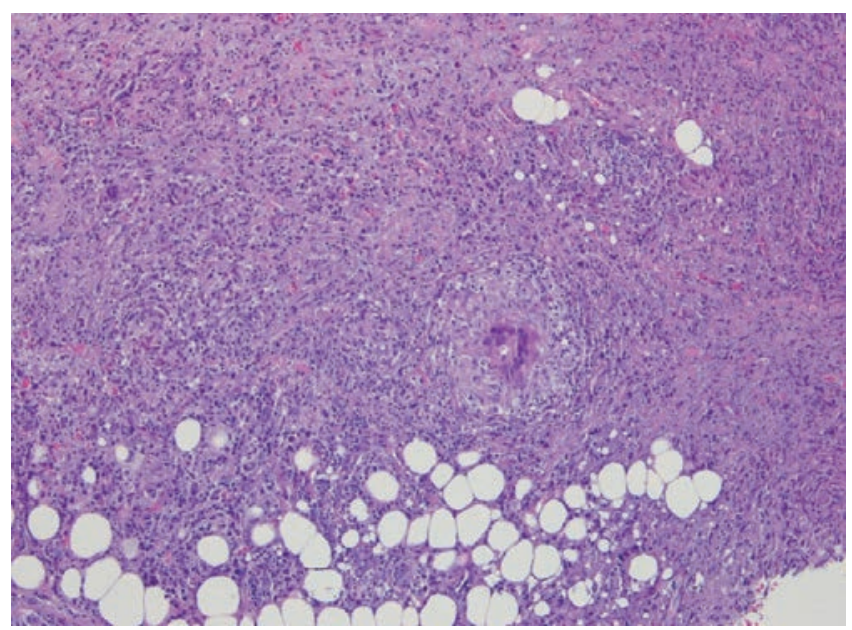

Figure 2. Histology of the left sciatic nerve. Fibroadipose tissue with inflammatory infiltrate with necrotic granuloma and multinucleated giant cells (H\&E stain, 100x). 


\section{REFERENCES}

1. Gomez-Reino JJ, Carmona L, Valverde VR, Mola EM, Montero MD; BIOBADASER Group. Treatment of rheumatoid arthritis with tumor necrosis factor inhibitors may predispose to significant increase in tuberculosis risk: a multicenter active-surveillance report. Arthritis Rheum 2003;48:2122-7.

2. Keane J, Gershon S, Wise RP, Mirabile-Levens E, Kasznica K, Schwieterman WD, et al. Tuberculosis associated with infliximab, a tumor necrosis factor alpha-neutralizing agent. $\mathrm{N}$ Engl J Med 2001;345:1098-104.
3. Duarte R, Campainha S, Cotter J, Rosa B, Varela P, Correia A, et al; Portuguese Society of Pulmonology; Portuguese Society of Gastroenterology; Portuguese Society of Dermatology and Venereology; Portuguese Society of Rheumatology. Position paper on tuberculosis screening in patients with immune mediated inflammatory diseases candidates for biological therapy. Acta Reumatol Port 2012;37:253-9. 